\title{
LA MULTIPLICATION DES PERSPECTIVES AU PROFIT DE L'EXPRESSIVITÉ: UNE ÉTUDE DU ROMAN LES SIGNES PARMI NOUS DE C.F. RAMUZ
}

\author{
Siba BARKATAKI \\ Université Jawaharlal Nehru, Inde \\ sibab2010@gmail.com \\ Article reçu le 12 mars 2016 | révisé depuis le 24 avril 2016 | accepté le 25 juin 2016
}

\begin{abstract}
RÉSUMÉ. Nous analyserons l'incertitude des voix et la création idiosyncratique des subjectivités provisoires dans le discours narratif chez Ramuz. L'analyse portera sur les diverses modalités liées au décentrement du discours narratif : la désorganisation des temps verbaux, l'emploi du pronom «on», du discours indirect libre ainsi que l'intertexte biblique. Nous étudierons également la notion de perception chez Ramuz. La simultanéité des perceptions contribuant au problème de détermination de la voix narrative met en relief l'exotopie de l'auteur par rapport aux personnages, ce qui relève du besoin inhérent de donner aux personnages leur propres voix. La multiplication des points de vue crée une collectivité de perspectives. Cette collectivité devient la conscience globale du texte qui s'achève avec l'interprétation du lecteur. Le rôle fondamental du lecteur se trouve ainsi souligné.
\end{abstract}

Mots-clés: conscience, intertextualité, perceptions, pluralité, voix.

\begin{abstract}
We will analyze the uncertainty of the voices and the idiosyncratic creation of provisonal subjectivities in the narrative discourse of Ramuz. The analysis will deal with the different modalities linked with the decentralisation of the narrative discourse : disorganisation of verb tenses, the use of the pronoun " on », free indirect speech as well as biblical intertext. We will also study the notion of perception in Ramuz's work. The simultaneous nature of the perceptions contributes to the problem of determination of the narrative voices that highlights the exotopy of the author vis-a-vis the characters, which indicates the inherent need of giving the characters their individual voices. The multiplication of the points of view creates a collectivity of perspectives. This collectivity becomes the global conscience of the text that is achieved with the interpretation of the reader. The fundamental role of the reader thus needs to be highlighted.
\end{abstract}

Keywords: conscience, intertextuality, perceptions, plurality, voices.

\section{INTRODUCTION}

Dans la présente étude nous analyserons la confrontation de multiples voix qui s'expriment face à l'imminence de l'apocalypse. La multiplication et la valorisation des points de vue mènent à l'éclatement de la position de l'instance narratrice. Cette dernière ayant désormais un statut polyvalent occupe divers positions dans le récit, tout en restant anonyme afin d'autonomiser les perceptions plurielles des personnages. 
L'histoire raconte une journée extraordinaire dans la vie des gens d'un petit village en Suisse qui autrement menaient une vie paisible sans incident. L'arrivée de Caille, un colporteur biblique, dans le village met en marche l'intrigue. Ses brochures annonçant la fin $\mathrm{du}$ monde déclenchent la peur dans le village et rendent les villageois sensibles aux signes avertissant l'imminence de l'apocalypse. De ce fait, même les simples éléments de la nature contribuent à construire un paroxysme qui alimente l'intrigue. Le titre du roman Les signes parmi nous soulève justement cet aspect apocalyptique qui transforme des éléments habituels en signes prédisant la mort et la destruction de l'humanité. "Cependant les choses autour de nous ne sont pas silencieuses; elles ont un message à nous transmettre, elles aussi" (Ramuz, 2005, p. 1233). Le pronom sujet «nous » signifie non seulement la communauté d'homme mais la dépasse pour inclure également l'auteur et le lecteur. Ce rassemblement d'homme, un aspect singulier de l'esthétique ramuzienne, devient la source de la polyphonie.

Cette étude se divise en trois parties. Dans un premier temps, nous déconstruirons l'univers unifié du roman au profit des perceptions différentes qui s'y expriment. Cette démarche permet de singulariser les différents points de vue afin de mettre en évidence la confrontation entre eux et révéler l'hétérogénéité inhérente de la communauté d'homme. Dans un second temps, il s'agirait d'analyser les diverses modalités liées au décentrement du discours narratif. L'analyse de la pluralité de voix reste inachevée sans étudier le rôle que joue le narrateur vis-à-vis des univers pluriels qui se manifestent dans le roman. Finalement, l'analyse portera sur la correspondance entre l'histoire inventée, la bible et la guerre. L'intertextualité nous révèle la façon dont l'auteur fait interagir l'actualité et l'apocalypse.

\section{MULTIPLICATION DES PRISES DE CONSCIENCES CHEZ RAMUZ}

D'après le dialogisme de Bakhtine, tout dialogue ou discours s'oriente vers d'autres discours et se manifeste comme pluralité de voix. La polyphonie est l'utilisation romanesque du dialogisme (Bres et Nowakowska, 2007, p.103). Dans le discours ramuzien nous témoignons de la polyphonie par la présence d'une multiplicité de voix anonymes. Il nous paraît opportun donc d'étudier la dimension discursive chez Ramuz et d'analyser l'interdiscursivité dans le roman qui s'inscrit dans le texte par le moyen d'interaction entre les voix diverses. Il s'agit d'un processus de communication qui comporte un éclatement du sujet, d'où émergent les multiples consciences. La pluralité de voix représente en réalité une pluralité d'idéologies qui se confrontent et qui fait preuve de la nature hétérogène de la communauté ramuzienne. L'individu est oblitéré et il est remplacé par la voix d'une conscience. L'absence du héros est ainsi significative car désormais toutes les voix entendues dans l'espace textuel sont mises sur un pied d'égalité. Dotée d'une telle autonomie d'expression inégalée, ces divers univers idéologiques se combinent dans l'unité de l'événement de la fin du monde. Vue de façon inverse, ce dernier se décompose en des traits de pensées différents. Dans le roman nous avons repéré trois consciences distinctes : le paysan-poète, la conscience apocalyptique et le paysanrebelle.

Le premier univers est celui où l'on entend la voix du paysan-poète anonyme. Ce dernier se contente pleinement de sa vie banale dans son pays. Cette voix innocente se doutant de peu réhabilite l'optimisme dans le roman. Ce paysan valorise sa vie dans le village en décrivant la beauté du cadre naturel au sein duquel il vit: "Avec du mélilot, avec des touffes de mille-pertuis, avec des beaux coquelicots rouges; et, parmi tout cela, une petite maison montée sur roues" (Ramuz, 2005, p.1235). Ce paysanpoète oppose la vision sombre de l'apocalypse à l'idée d'une régularité et continuité paisible de la vie: "Est-ce qu'on distingue les Signes? quels Signes y aurait-il ici? On ne voit que tranquillité, vérité, régularité. [..]" (Ramuz, 2005, p. 1252).

Ici s'installe le paradoxe. D'un côté, cette voix se réjouis de la banalité de sa vie terrestre, de l'autre, tout comme les prédictions apocalyptiques, elle tire le texte 
vers le cosmique. Or, contrairement aux prédictions, pour le paysan-poète il s'agit d'un destin heureux favorable à la vie. A cette fin, la voix anonyme ironise le discours par le moyen de l'anthropomorphisation de la nature. Alors que les signes de l'apocalypse saisissent toute la scène, la nature fait entendre sa voix à travers le paysan pour se moquer des nouvelles d'apocalypse. Ce procédé esthétique fait également preuve de la symbiose harmonieuse du paysan avec la nature.

[..] la Parole une fois de plus était mise en circulation.

Cependant les choses autour de nous ne sont pas silencieuses; elles ont un message à nous transmettre, [..] elles sont une réunion qui dit: «On est là ; regardez-nous. » [..] La fontaine, d'une voix monotone et sans fin, répète [..] : " On est, on coule, je fais beau, [..] je coule, l'herbe m'aime, l'herbe a besoin de moi ». (Ramuz, 2005, p. 1233)

Bien que ces instances de communication soit le fruit de l'imagination $\mathrm{du}$ paysan, elles ont une telle force qu'elles acquièrent une forme tangible dans le récit. Le dialogue entre l'homme est la nature est la preuve d'un grand accord entre eux "où on est sûr de mutuellement se connaître" (Ramuz, 1970, p.156). C'est grâce à cette symbiose que le paysan-poète reste tranquille face au chaos de la fin du monde: "Comme tout est tranquille pourtant dans ce pays d'ici où rien ne semble avoir changé depuis toujours, [..] L'homme et le pays sont ici en étroite correspondance ; [..] C'est ce qui rassure, c'est ce qui est beau". (Ramuz, 2005, p. 1249)

Une telle sublimation de la vie terrestre a également pour but la remise en question de l'implication du message biblique dans l'actualité. Etant donné que le colporteur biblique fait circuler le message de l'apocalypse et par là, devient la source du chaos, en critiquant Caille, la voix du paysanpoète critique le détachement total et l'indifférence de la conscience biblique pour la vie, et pour les malheurs d'autrui: "Il (Caille) repassa sous les platanes; ils disaient : «On est beaux, regardez-nous »; il ne les a pas regardés" (Ramuz, 2005, p. 1234). En effet, le texte est ponctué de cette idée d'indifférence de la conscience biblique envers la vie humaine. Elle est également transposée au plan du discours par la voix du paysan-poète : "[..] mais c'est qu'on s'est défait des choses de la terre, alors peut-être bien que les choses de la terre, elles aussi, se sont désintéressées de vous, et le soleil terrestre n'a plus d'influence sur vous" (Ramuz, 2005, p. 1266). Quoique cette voix soit de plus en plus dominée au cours de l'histoire, quand les signes apocalyptiques se multiplient, à la fin du roman, c'est cette voix $\mathrm{du}$ simple paysan-poète qui triomphe. Sa voix s'élève de nouveau lors du dénouement pour célébrer le recommencement de la vie dans le monde. "[..] que ceux qui sont avec leurs morts pleurent leurs morts; ici on veut vivre avec les vivants. [..] Ça va comme toujours » ; - recommencement" (Ramuz, 2005, p. 1311).

Il n'en est pas moins que tout au long du roman cette voix est confrontée et neutralisée par plusieurs autres voix qui participent au mouvement paroxystique de l'histoire. L'une de ces voix se distinguant nettement de celle du paysan-poète et celle de la conscience apocalyptique. Cette deuxième conscience déchiffre les événements de la journée qui constitue l'unité de temps du roman, et y voit l'inéluctabilité de l'Apocalypse. Cet univers idéologique se déploie par le biais des signes apocalyptiques dans l'espace textuel. Cette conscience se compose de plusieurs voix dans le roman. Il y a tout d'abord la voix soucieuse d'un petit groupe de paysans, frappé par le malheur, qui cherche des explications dans les prédictions pour donner l'expression à ses soucis: « Peut-être que c'est vrai, peut-être que ce qu'il annonce est vrai, peut-être bien qu'il a la connaissance des choses" (Ramuz, 2005, p. 1261). Et ailleurs, "...ces famines, cette mortalité, ces maladies sont signes, d'après le livre, que la fin $\mathrm{du}$ monde n'est plus tellement éloignée [..]" (Ramuz, 2005, p. 1246).

Or, de tels propos parsemés dans le texte accordent "l'effet de réel" (Barthes, 1982, p.81) à l'Apocalypse. La reconnaissance des prédictions par le paysan en facilite la récupération dans le tissu narratif. Ensuite, il y a la voix de ceux comme Caille, le 
colporteur biblique, dont la vie se détermine entièrement par le message de la bible et par sa mission de faire propager ce message. Bien que Caille soit le seul personnage dans le roman qui apporte le message de l'apocalypse dans le village, sa voix s'unifie à celle de toute la communauté de croyants cherchant à persuader les gens de la vérité des Ecritures. Ses pensées sont extériorisées et élevées au niveau d'idéologie par le narrateur. Caille devient par-là, la porteparole de la communauté de croyants: "Notre métier à nous est de frapper à toutes les portes, surtout à celles qui ne s'ouvrent pas [..]" (Ramuz, 2005, p. 1232).

$\mathrm{Au}$ début $\mathrm{du}$ roman, Caille est l'élément déclencheur de la conscience apocalyptique. Son arrivée dans le village met en marche la réalisation de l'Apocalypse : "quand même les Signes sont venus, et s'annoncent de toute part, mais moi je ferai éclater les Signes à leurs yeux" (Ramuz, 2005, p. 1232). Or, à mesure que l'histoire progresse, cette voix s'atténue et cède la place à la voix même du message biblique. Ce message de l'Apocalypse, qui se distingue de la voix du messager, envahit au fur et à mesure l'espace textuel et y introduit le temps biblique. Il apporte non seulement un symbolisme distinctif dans le texte, mais il modifie également le langage, le ton et le rythme $\mathrm{du}$ discours. Le temps biblique se fusionne avec la diégèse à tel point qu'on a l'impression que "les Temps d'aujourd'hui ressemblent à ces premiers Temps" (Ramuz, 2005, p. 1234).

[..] il se trouve que les Temps se sont repliés sur eux-mêmes, la Fin ressemble au Commencement.

Les Temps de la parole proférée et les temps de la parole réalisée sont en ressemblance et en voisinage [..]. (Ramuz, 2005, p. 1263)

Ce morceau ci-dessus du livre de l'Apocalypse est soigneusement tissé dans le texte sous une forme narrativisée pour qu'il ait une apparence naturelle au sein du cadre campagnard.

L'une des astuces adoptée pour diffuser la voix biblique, quoique distinguée, dans la parole paysanne, est de la mettre en situation dialogique avec $\mathrm{d}$ 'autres instances du roman. Prenons comme exemple cet extrait du roman où le message de l'Apocalypse est mis en scène en quatre temps : tout d'abord une annonce, ensuite le message de l'Apocalypse, puis l'instance narratrice, enfin la voix anonyme d'un paysan témoin de la maladie de son confrère. Cette dernière voix se distinguant par un « je » peut aussi être attribuée à l'auteur qui se présente comme instance textuelle :

L'homme fut frappé, pendant qu'il aiguisait sa faux [..] Il tomba assis, pencha de côté ; sa figure devint toute blanche; il ouvrait sa bouche, montrant ses dents [..]

Il est dit que les maladies viendront, et ce sera le Temps du quatrième sceau. Alors le Cheval Pâle est lâché, le Cheval Pâle frappe d'en haut. [..]

Est-ce qu'on a vu ce nuage ? ou bien si les yeux de chair ne suffisent pas pour le voir? Mais, moi, je l'ai vu, il venait ; il a passé par-dessus la crête ; c'est ce qu'il y a de plus robuste qui est frappé; les Temps sont là de la mortalité [..]. (Ramuz, 2005, p. 1236)

L'instance de la maladie du paysan est juxtaposée au morceau de l'Apocalypse annonçant l'avènement des maladies. Bien qu'il n'y ait pas de dialogue entre les deux premières scènes la reprise du thème de la maladie et du verbe "frapper » créés un lien causal entre eux. Cette juxtaposition a pour but de mettre en évidence la réalisation de la prophétie. Ensuite, le texte biblique est suivi de deux phrases de transition : "Est-ce qu'on a vu ce nuage ?...». Ces phrases en style indirect libre permettent le retour au contexte du roman. De surcroît, en invitant une réflexion sur la prédiction à laquelle elles succèdent, ces phrases interrogatives valorisent le message et assurent un passage harmonieux du texte biblique au roman. Finalement, la séquence se termine par la justification de la vérité du message. L'affirmation de la présence du Cheval Pâle dans le village: «je l'ai vu», par un paysan anonyme ou par l'auteur qui se pose comme sujet perceptif, rend légitime et réel le constat de la conscience biblique. En vertu d'être aperçu par une instance textuelle, le message se matérialise et se transmet dans la réalité diégétique. En outre, le verbe «frapper»se 
présentant une troisième fois sert à créer un effet d'homogénéité dans toute la séquence. Cette dernière se trouve ainsi récupérée par la logique du texte.

La troisième conscience qui se fait remarquée dans le roman est celle du paysan-rebelle Cette voix se distingue de la voix romantique du paysan-poète par son ancrage dans l'actualité, qui lui fait gagner en pragmatisme, mais surtout dans la mesure où elle s'oppose ouvertement à la présence provocatrice de Caille et de la conscience apocalyptique dans le village : "Tu le connais, laisse-le! C'est le pâle qui nous intéresse. Il faudrait lui régler son compte, parce que c'est des gens qui portent malheur [...]" (Ramuz, 2005, p. 1281). Et, "Allez-vous en, je vous dis! Laissez-nous tranquille! Comme s'il n'y avait pas déjà assez de malheurs !... " (Ramuz, 2005, p. 1282). Epanoui et sûr de son vécu, cette voix refuse d'accepter l'interprétation apocalyptique des signes. Elle y voit au contraire une causalité et rapporte les maladies, la chaleur et les morts à la guerre, à l'industrialisation et aux problèmes sociaux: "Traîneurs de terre à vos souliers, profitez pendant que vous pouvez !" (Ramuz, 2005, p. 1256). Et ailleurs, "[..] maudite idée qu'on a eue d'autoriser cette fabrique à s'établir chez nous ! [..] On a de la peine à respirer [...]" (Ramuz, 2005, pp. 12781279).

Grâce au scepticisme qu'elle apporte au roman, l'espace textuel devient un lieu de confrontation entre l'actualité et l'apocalypse. Toute tentative d'installer le temps biblique est confrontée par l'évocation des thèmes historiques rétablissant le temps humain, à savoir, les conflits sociaux, les besognes quotidiennes, la grève et la justice sociale. Le paysan-rebelle oppose le côté social de la vie terrestre à l'aspect céleste de la vision apocalyptique :

Le Livre ne s'occupe pas des personnes. Tout au plus, y est-il fait allusion à Sept Rois, et le dernier n'est point venu encore; quand il sera venu, dit le Livre, il ne durera qu'un peu de temps. [..]

«Eh bien! tant mieux s'il ne dure pas ; nous autres, on est républicains. »

(Ramuz, 2005, p. 1240)
Donc, toutes ces trois consciences représentent le moyeu fixe de la polyphonie dans le roman. Il faut également remarquer que ces trois voix ont la primauté par rapport au narrateur. Le rôle explicatif et descriptif de l'instance narratrice est limité. L'histoire se construit par la voie de l'interdiscursivité. Dans un tel éthos discursif, le narrateur se rend au service des voix différentes afin qu'elles puissent mieux s'articuler. En effet, le narrateur joue un rôle polyvalent voire ambigu car il n'y a aucun parti pris. Cette adhésion impartiale à toutes les voix accordent à ces dernières un statut égalitaire dans l'espace textuel.

\section{LE NARRATEUR RAMUZIEN FACE À LA PLURALITÉ DE VOIX}

L'étude sémantique des voix relève trois dimensions de la vocalité : la dimension corporelle: la voix comme caractéristique physique, la dimension discursive: la voix comme discours, et la dimension narratologique : la voix en tant que terme de sémiotique narrative (Bres et Nowakowska, 2007, p.105). L'analyse des voix qui s'expriment dans l'espace textuel ramuzien n'est pas complète sans analyser la dimension narratologique c'est-à-dire, le rapport que la voix narratrice entretient avec les autres voix du texte. Il va sans dire que le narrateur fait partie intégrale du roman ramuzien. Or cette voix est un élément complexe. D'après Bakhtine la voix narratrice "offre la singularité d'être bivocal. Il sert simultanément à deux locuteurs et exprime deux intentions différentes : celle-directe du personnage qui parle, et celle - réfractée - de l'autre. Pareil discours contient deux voix, deux sens, deux expressions. En outre, les deux voix sont dialogiquement correlates" (Bakhtine, 1978, p. 145). De même, chez Ramuz, la voix narratrice devient la source de la polyphonie en vertu de sa coexistence avec toutes les autres voix du récit. A la fois partisan et détaché, le narrateur fait preuve d'une alternance des points de vue et d'une polyphonie mêlée. Existant dans le récit la plupart du temps par l'entremise du pronom " on ", le narrateur voyage dans le récit entre les différentes voix ainsi qu'entre les divers niveaux narratifs. 
En générale, la voix du narrateur ramuzien ne se distingue pas de celle des personnages, à l'exception de quelques rares moments comme celui dans l'incipit où elle occupe un point de vue omniscient lorsqu'elle annonce l'arrivée de Caille dans le village : "Caille, le colporteur biblique, a suivi encore un moment la route qui longe le lac ; puis s'est engagée dans un chemin de traverse" (Ramuz, 2005, p. 1231). Le narrateur aperçoit le colporteur bien avant les autres personnages. Le syntagme nominal «le colporteur biblique» et le complément adverbial de temps «encore un moment» dans la toute première phrase du roman indique que le narrateur le connaît et le surveille depuis un bon moment. Cela accorde une antériorité à la voix narrative par rapport au temps fictif. Le narrateur domine et dirige la perception du lecteur au profit du récit. Une telle prise de position au tout début, resserre le texte car il introduit directement, sans beaucoup d'explications, le déclencheur d'intrigue dans le cadre fictif.

Or, au cours de l'histoire, l'instance narratrice abandonne cette position privilégiée pour prendre une position plus ambiguë : "on devinait le lac, plus qu'on ne le voyait, [..]" (Ramuz, 2005, p. 1231). La vue du narrateur s'identifie à celle d'un paysan anonyme, qui connaît bien le paysage et les habitudes du village. Ce point de vue externe non seulement rend la narration neutre et objective, mais il rend le récit plus imagé. N'ayant plus l'accès à l'univers mental des personnages le narrateur décrit leurs gestes pour faire part de leurs sentiments : "Il a levé le bras au-dessus de sa tête pour dire: «Foutez-moi le camp!» [..] Rien à faire ; Caille soupire" (Ramuz, 2005, p. 1235).

En effet, c'est la mobilité du narrateur dans le texte qui fait éclater les points de vue. D'un côté, le narrateur en tant qu'observateur, veut autonomiser les personnages par le biais du gestuel, de l'autre, il plonge dans le monde intérieur de Caille pour extérioriser la conscience biblique. Le colporteur est un personnage complexe. A l'intérieur de sa personne timide existe une force de conviction qui métamorphose tout le village en une scène d'apocalypse. La focalisation interne fait éclater la conscience apocalyptique qui demeure en lui et dévoile l'absolutisme de cette vision. Cette dernière domine le colporteur à tel point qu'il lamente lui-même son insignifiance : "n'étant rien moi-même, ou n'étant que son instrument [..]" (Ramuz, 2005, p. 1232). L'absence d'originalité permet au narrateur d'universaliser la vision de Caille. La voix de sa conscience devient la porte-parole de la communauté de croyants : "Il y en a qui sont portés par l'Esprit à la compréhension des Prophéties, [..] notre métier, à nous, n'en est pas moins d'aller de porte en porte" (Ramuz, 2005, p. 1232). Dans cette citation il s'agit d'une focalisation interne, mais le point de vue est exprimé par le pronom sujet «nous » qui sert à effacer la subjectivité d'un énonciateur individuel. Ce pronom signifiant l'unité du groupe sert à englober Caille, la communauté de colporteurs ainsi que la voix narratrice.

Ces différentes postures du narrateur ouvrent de multiples espaces et de multiples niveaux narratifs dans le récit. Le narrateurtémoin au niveau diégétique, qui d'ordinaire ne porte pas de jugement sur les personnages, se déplace de temps en temps au niveau extradiégétique pour les commenter et juger: "C'est l'explication des événements actuels à la lumière des Ecritures. [..] les Temps sont proches. [..] Il ne disait pas tout cela, [..] étant seulement le marchand de la chose, faisant commerce de la chose [..]" (Ramuz, 2005, p. 1232). Le narrateur hiérarchise le récit et prend une distance du colporteur qui, d'après lui n'est pas suffisamment doué pour interpréter la bible. Ces propos du narrateur "Il ne disait pas tout cela [..]" ouvrent un nouvel espace dans le récit. Celui où le message se distingue du messager. Le message biblique commence à avoir une présence indépendante (au titre de personnage) par rapport au messager. Or, cette voix ne se dissocie pas complètement de la voix narratrice et s'y mêle à plusieurs reprises. Ce phénomène fait preuve de la polyphonie mêlée fréquemment employée dans le roman. Il s'agit de "deux voix qui se superposent, mais dans une confrontation qui pourrait sembler être d'égalité" (Nølke et Fløttum, 2004, p.156). En fait, dans l'œuvre il 
est parfois difficile voire impossible de distinguer entre la voix narratrice et celle des personnages: "S'il n'est pas crié en nous, l'encouragement, compte-t-il ? [..] l'Esprit m'a-t-il abandonné ?" (Ramuz, 2005, p. 1265). Cette instance de focalisation interne de Caille englobe trois voix : celle de Caille, d'un verset biblique et d'une collectivité anonyme représentée par «nous ».

Dans un tel lieu d'expression de multiples locuteurs, le récit se divise en plusieurs couches d'énonciations et en des zones de transitions incertaines représentées par le discours indirect libre. Comme la plupart du temps il s'agit d'un énonciateur anonyme appartenant à une collectivité, l'on ne peut pas attribuer des énoncés à un sujet d'énonciation spécifique. Le narrateur se représente dans le récit par le pronom " on » indéterminé et impersonnel. Par le biais de ce pronom il reste à la fois subjectif, détaché ou passif, n'ayant pas un point de vue fixe. Le jeu entre la voix d'un personnage et le narrateur étant très serré, le «on » permet au narrateur de se déplacer d'une prise de conscience à une autre, c'est-à-dire, d'un énonciateur à un autre. Le "on" ramuzien comporte ainsi un double caractère: nonpersonne et personne: "Ils disent: C'est ce grand fou avec son livre et ses histoires; qu'ils n'y reviennent pas! Mais aussi pourquoi l'a-t-on écouté ? " (Ramuz, 2005, p. 1309).

En outre, le « on» ramuzien avec des onomatopées qui parcourent le texte ainsi que les présentatifs « c'est » et « il y a » ont la capacité de rendre compte de l'actualité de l'expérience vécue. En plus, ils soutiennent bien le rythme serré du récit y apportant une urgence. Ils contribuent donc à oraliser le récit car ce dernier fait preuve d'une immédiateté qui est typique des textes oraux. L'oralité du récit dans le roman est une marque polyphonique importante car il s'agit de valoriser le discours des personnages au détriment de la péripétie. D'après Mahrer, (2006, p.228) le pronom «on» et les présentatifs fonctionnent comme des «embrayeurs de discours» qui transposent la narration du plan de l'histoire à celui de discours. En outre, le narrateur qui s'impose par la voie de ces embrayeurs adopte entièrement la langue et le point de vue des personnages.

Par ailleurs, le pronom «on» chez Ramuz, aide à maintenir l'anonymat inhérent des points de vue, ce qui d'ailleurs permet la construction d'un univers au sein duquel s'articule une multiplicité de perceptions indépendantes. En fait, le rôle impersonnel du narrateur met en évidence la pluralité de prises de perception.

Comme l'histoire se construit sur une base discursive et les événements se déroulent en une journée. L'unité de temps $\mathrm{du}$ roman impose une immédiateté à la perception de ces événements. Les personnages se rendent compte subitement de la gravité de leur situation. En une journée, le village va d'un calme habituel au chaos absolu. Le désordre et la précipitation se font remarqués surtout dans la manière dont les personnages aperçoivent leur réalité concrète. Par conséquent, dans la composition du texte, les voix se succèdent tantôt, d'après une suite logique et tantôt, de manière spontanée comme une exclamation ou une conséquence immédiate. L'histoire se raconte du point de vue de "l'expérience perceptive" (Mahrer et Wiser, 2007, p.216) des personnages. Ces prises de positions s'enchaînent ou parfois, se juxtaposent sans rapport les unes avec les autres, afin d'imiter la simultanéité des événements.

Vers les 3 heures et demie, à présent; est-ce qu'on ne sent pas qu'on commence à n'y plus tenir ?

Ceux qui parlaient beaucoup ne parlent plus [..].

Les femmes sont devenues sujettes à des malaises.

Il y a un char à bancs qui attend devant chez Aloys; le cheval brille dans l'ombre [..] il ne se défend plus contre les mouches, [..].

C'est qu'ils ont été cherché partout un médecin, le nôtre vient de mourir ; [..]. (Ramuz, 2005, p. 1277)

Les différentes postures de l'instance narratrice ci-dessus traduisent "la mobilité du récit dans sa dimension spatio-temporelle" (Kila, 2013, p.76). En fait, le remaniement de la position de l'instance narratrice et l'utilisation de multiples temps verbaux accordent à la narration un effet 
cinématographique. L'auteur imite le mouvement de la caméra pour avoir une vue panoramique de la scène. La prise de vue d'un personnage est interrompue par d'autres perceptions et l'auteur fait un montage de divers plans pour produire une image globale. Ceci rompt la narration monotone et lui accorde une immédiateté théâtrale en verbalisant la perception et la transformant en action. Au niveau de langue, ces ruptures énonciatives constituent la polyphonie énonciative. Par ailleurs, chaque image évoque un sentiment et une sensation particulière. La juxtaposition de ces images implique la pluralité de vues et contribue à la création d'une scène riche en images et sentiments.

\section{L'INTERTEXTUALITÉ CHEZ RAMUZ}

Pour élargir le cadre conceptuel de l'interdiscursivité et du croisement de voix et de perception, il serait impératif d'étudier l'interaction entre l'histoire fictive et d'autres textes. Les Signes parmi nous est en effet, un mosaïque de plusieurs textes qui se transforment et s'organisent au sein de l'espace textuel. Cette "absorption et transformation" (Kristeva, 1969, p.85) d'autres textes forme la base primordiale de la polyphonie dans le roman. Ramuz y fait interagir deux thématiques principales: la guerre et l'Apocalypse de Jean. Ces deux thèmes qui entraînent l'idée de la mort et de la destruction apportent à l'histoire inventée l'urgence, le chaos et le désespoir nécessaires pour créer un univers qui se trouve au seuil de l'annihilation. Tandis que la guerre remplit une fonction synchronique en inscrivant l'histoire inventée dans l'actualité de l'époque; l'intertexte apocalyptique accomplit une fonction diachronique voire mythique: les prédictions ayant la qualité inhérente de rendre un événement perpétuellement possible.

Les références à l'actualité quotidienne indiquent la présence de la Première Guerre comme intertexte significatif. Sa présence y est discursive. Elle se trouve appropriée dans les propos des personnages: "Eh bien! il paraît qu'à présent vous êtes fameusement montés en fait d'artillerie [..]" (Ramuz, 2005, p. 1245). Dans ces citations, Ramuz parle du développement considérable de l'artillerie durant la Première Guerre en 1914. En effet, les articles et les communiqués des quotidiens de l'époque ont servi comme intertextes importants rapportant la réalité concrète à l'écrivain qui se sert de la guerre comme toile de fond.

L'interprétation ambivalente de la guerre forme la base du dialogisme dans le roman. Les renvois implicites à la guerre contribuent à exposer des aspects importants de la nature humaine. D'un côté on lamente la perte de la vie humaine, la destruction et la violence exercée par l'homme, de l'autre, on valorise la création de nouvelles normes juridiques, morales et sociales qui fait preuve de l'intelligence et de la grandeur humaine. La thématique de la guerre constitue en effet «le dialogisme interne» qui se manifeste "dans le plan de l'énoncé de la constitution dialogique du discours" (Magureanu 2014, p.248).

Ramuz juxtapose plusieurs documents et points de vue dans l'espace dialogique afin de faire dialoguer les différentes perspectives sur la guerre, qui ne soient pas d'ailleurs assujettis à une voix unificatrice. Les divers points de vue mettent en scène cette ambivalence inhérente à la guerre. Dans un premier temps, les nouvelles de la mort des milliers des gens de l'épidémie de grippe espagnole rapportée par la Gazette de Lausanne du 1918, contribuent à mettre l'incertitude et la peur au premier plan. Les pires conséquences de la guerre notamment les fluctuations de la monnaie, le mécontentement et la peur de l'avenir, sont bien ressenties sans que l'auteur les situe dans une réalité historique.

On dit que le mark est à 66 et partout la puissance d'achat de l'argent a diminué au moins de la moitié [..]

On dit qu'il y a des famines partout dans le monde [..]

On dit également qu'il y a beaucoup de maladies dans le pays, [..] (Ramuz, 2005, p. 1238)

Dans un second temps, la nature absurde de la guerre et ses contradictions sont mises à lumière. Ces contradictions se situent dans le contexte de la neutralité de la Suisse pendant la guerre. En réalité, Ramuz prend une position politique contre la 
neutralité de la Suisse. Dans le roman, il évoque la situation paradoxale de vivre très proche de la guerre, et pourtant de ne pas la vivre. Un ton ironique parcourt le texte qui se moque de la neutralité. Ainsi une altérité permanente traverse le discours où le sentiment de soulagement est remplacé par celui d'impuissance :

Dans d'autres pays, du moins, quand ils meurent, on sait pourquoi, on sait comment, leur mort sert à quelque chose ; [..] c'est pour leur pays qu'ils meurent, ils voient les ennemis s'en aller de chez eux, mais les nôtres? (Ramuz, 2005, p. 1254)

Chez Ramuz la guerre de 1914 ainsi que la révolution russe de 1917 font éclater des perceptions car malgré la mort qu'elles entrainent, elles sont projetées comme étant des phénomènes exemplaires, du fait que ces deux événements font preuve de la collectivité humaine. L'auteur y voit naître «le peuple» (Froidevaux, 1982, p.160) et de nouvelles normes morales et sociales : "Il n'y a de culture que dans la souveraineté" (Ramuz, 1967, p.29). Ce peuple est prêt à «réaffronter» la réalité première de la vie sans se soumettre aux enseignements, aux règles et aux conventions qui lui ont été imposés auparavant.

Dans le roman, la révolution russe de février 1917 présente le modèle d'un soulèvement spontané du peuple. Ce dernier dirigé par la chair et le cœur, devient la force vitale de la révolution dans le roman.

C'est ça ! rester les bras croisés ; bonne affaire pour les bourgeois !... Moi, je suis pour la justice qu' on se fait soimême. On en a assez des cadeaux de votre bon Dieu !...»[..] «On fera la révolution. » (Ramuz, 2005, p. 1242)

Pendant la période de la guerre, on assiste à l'apparition de l'homme ramuzien sensible aux signes de la nature. En réalité, la guerre lui fait nourrir l'espoir de la disparition totale du monde de l'avantguerre et la renaissance d'un monde régi par le cœur, l'instinct, la solidarité, et bien d'autres concepts. Ramuz traduit cette vision d'un nouvel ordre dans ce roman où l'on voit un monde sensible et intuitif qui laisse déceler des signaux prophétiques de la destruction. La mort suivie de la renaissance d'un nouveau monde régi par un nouvel ordre accorde un ordre cyclique au roman. La guerre devient ironiquement la promesse d'une nouvelle vie.

$$
\begin{aligned}
& \text { J'ai peur. } \\
& \text { Peur de quoi ? } \\
& \text { Crois-tu que c'est la fin du monde?» } \\
& \text { Mais il rit et il dit: } \\
& \text { "C'est le recommencement du } \\
& \text { monde» (Ramuz, 2005, p. 1305). }
\end{aligned}
$$

Ainsi paradoxalement, la thématique de la guerre apporte au premier plan l'idée de la continuité de la vie. L'intrication de la guerre et des prédictions de l'Apocalypse accorde une dimension cosmique à cette idée esthétique de Ramuz.

Une étude génétique $d u$ roman indique que Ramuz appuie pleinement sur la Bible, plus spécifiquement sur la version d'Ostervald de 1772. Ses diverses notes préparatoires montrent que l'auteur choisit particulièrement les séquences dites septénaires. La chaleur caniculaire, l'orage, les instances de mort, le tremblement de terre sont autant d'éléments qui accordent au récit un mouvement paroxystique. Ainsi l'auteur choisit-il des éléments de nature romanesque qui sèment la terreur et l'angoisse :

Assemblez-vous, oiseaux, pour le
festin du grand Dieu, pour manger la
chair des rois, la chair des capitaines.
[..] Pour manger la chair des
Puissants, pour manger la chair des
chevaux et la chair de ceux qui les
montent [..] (Ramuz, 2005, p. 1303) Tous les éléments apocalyptiques ne servent qu'à transmettre le message que la fin est proche. Ce sentiment d'imminence parcourt le texte et construit une atmosphère d'urgence. Ramuz se rapproche de la vision de Jean proposant l'annihilation sans aucun espoir de vie. Le discours apocalyptique transforme le roman en une unité ouverte car ce discours se voit être envisagé "comme un perpétuelle devenir toujours renouvelé par le dialogisme" (Stolz, 2015). "Ça n'a été encore qu'un avertissement, Seigneur. Ta miséricorde est grande. Tu n'a pas voulu qu'aucun de ceux qui le peuvent manquent l'occasion de se repentir" (Ramuz, 2005, p. 1313). Ainsi avec la thématique de la Fin du monde Ramuz introduit un paradigme mythique car c'est un événement 
perpétuellement possible. Il n'en est pas moins que Ramuz s'écarte d'une manière très nette de l'idéologie religieuse. Il emploie la Bible à des fins purement esthétiques, ce qui d'ailleurs met en évidence la modernité de sa démarche artistique.

Le croisement du temps biblique et du temps fictif se réalise à plusieurs niveaux dans le texte: dans les descriptions, les propos des personnages, les allusions et les multiples positions de l'instance narratrice. Les versets bibliques se trouvent incorporés selon des modalités différentes. Quand il s'agit d'une citation littérale, l'écrivain la met en italique ou par l'usage typographique des guillemets. Il tisse également les versets dans la texture narrative sans aucune marque distinctive. L'incorporation des versets de la Bible dans un texte qui fait exprimer des paysans ordinaires crée un récit hybride, insolite et met en évidence le style novateur de l'écrivain. En tout, le tissage d'une histoire fictive, de l'actualité de la guerre et de l'intertexte biblique en un seul récit n'est que le fruit d'un immense travail de recherche, de correction et de remaniements de structure. Le vrai défi pour l'écrivain est de maintenir un effet d'homogénéité dans le texte tout en introduisant le vocabulaire, le ton, le rythme et les images bibliques dans un cadre campagnard dévoué d'imagination et d'expression. Or, Ramuz se sert de la Bible d'Ostervald connue pour ses déploiements symboliques et donc préférée des artistes. Quoi qu'il en soit, la correspondance entre le cadre campagnard et l'imaginaire biblique effectue un transfert de valeur esthétique en deux sens. En mettant le paysan face au monde de la bible, Ramuz esthétise son rapport au monde. Le paysage imaginaire du simple paysan se trouve ainsi élevé. En revanche, le monde austère de la bible se voit popularisé car il est accommodé aux banalités d'une vie paysanne.

\section{CONCLUSION}

Afin de réaliser une simultanéité de discours, l'auteur crée des situations où les gens se rassemblent. Force est de constater plusieurs scènes qui se déroulent dans l'Auberge ou dans la rue où les gens se regroupent et discutent ensemble, exposant de ce fait, les divers points de vue. La décomposition de la collectivité dans le roman fait apparaître trois univers idéologiques notamment, la conscience du poète, celle de la bible, et la conscience révolutionnaire chacun cherchant un moyen d'expression autonome. Alors que la conscience apocalyptique et la conscience du paysan-rebelle constituent deux pôles antagonistes mettant en relief l'opposition entre l'actualité et le cosmique, la conscience du paysan-poète représente le mi-chemin qui fait ancrer le cosmique dans la banalité de la vie quotidienne. Cette voix apporte aussi de l'optimisme là où persistaient le doute et le pessimisme : "Mieux vaut la pluie, huit jours du long, qu'une minute de grêlons" (Ramuz, 2005, p. 1312).

Par ailleurs, l'intertextualité accorde un contexte aux discours des personnages. De ce fait, leurs mots sont chargés d'un sens (religieux, historique ou social). En plus, la position ambigüe qu'adopte le narrateur visà-vis de la guerre et la prophétie est représentative de l'hétérogénéité constitutive du sujet.

Dans un tel carrefour de voix, l'auteur cherche lui aussi à trouver sa voix dans le texte à travers les différents déictiques: marque de la personne «je», «vous», le discours indirect libre ainsi qu'un ton ironique adopté lorsqu'il s'agit de la vision apocalyptique de Caille.

La présence de trois consciences différentes exprimées tour à tour par les personnages, le narrateur et parfois par l'auteur, fait que les Signes acquièrent des significations variées: la continuité paisible de la vie, la fin $\mathrm{du}$ monde et le recommencement d'un nouvel ordre. Ainsi le titre emblématique du roman Les Signes parmi nous, porte déjà en lui le grain de la polyphonie. Il est d'ailleurs laissé au lecteur, au titre d'interlocuteur, de déchiffrer cette ambigüité qui relève de cette hétérogénéité. Le pronom «nous » dans le titre du roman est donc symbolique car il désigne une collectivité constituée par les personnages, le narrateur, le lecteur et l'auteur. 


\section{REMERCIEMENTS}

Je voudrais remercier la Confédération Suisse pour la bourse de recherche qui a amélioré mon travail. Je tiens tout particulièrement à remercier $\mathrm{M}$. Frédérique Tinguely et Mme Sylviane Dupuis de l'Université de Genève pour tous matériels de recherche.

\section{RÉFÉRENCES}

Bakhtine, M. (1978). Esthétique et théorie du roman. Paris : Gallimard.

Barthes, R. (1982). Littérature et réalité. Paris : Editions de Seuil.

Bres, J. et Nowakowska, A. (2007). Voix, point de vue...ou comment pêcher le dialogisme à la métaphore. Cahier de praxématique, 49, 103-132. doi : 10.4000/praxematique.937.

Froidevaux, G. (1982). L'Art et la Vie, L'Ésthétique de C.F Ramuz entre le Symbolisme et les avant-gardes. Lausanne : L'Age d'Homme.

Kila, N. (2013). Dynamismes et structures de la perception dans l'cuvre de Charles Ferdinand Ramuz (Thèse de doctorat, Université d'Artois, France). Repéré à http://irodalomdoktori.btk.pte.hu/files/
tiny_mce/dolgozat.KILA.No\%C3\%A9 mi.pdf.

Kristeva, J. (1969). Sémiotikè, recherches pour une sémanalyse. Paris : Seuil.

Magureanu, A. (2014). Dialogisme, polyphonie et intertextualité dans le dialogue romanesque. Editura Academiei RRL, 3, 247-258.

Mahrer, R. (2006). Un français de plein air : la langue romanesque de C. F. Ramuz. Le français moderne, 2, 219-235.

Mahrer, R. et Wiser, A. (2007). La notion de temporalité phénoménologique chez C.F. Ramuz (Présence de la mort, 1919) et Claude Simon (La Bataille de Pharsale, 1969). Paris : Harmattan.

Nølke, H. et Fløttum, K. N. (2004). ScaPoLine - La théorie scandinave de la polyphonie linguistique. Paris : Editions Kimé.

Ramuz, C.F. (1967). Raison d'Être, CEuvres complètes 7. Lausanne: Éditions Rencontre.

Ramuz, C.F. (1970). Découverte du monde, Lausanne : Plaisir de Lire.

Ramuz, C.F. (2005). Les Signes Parmi Nous, Romans, tome 1, Bibl. de la Pléiade. Paris : Gallimard.

Stolz, C. (2015) Polyphonie et intertextualité. Repéré à www.fabula.org. 